\title{
Benzodiazepine Receptor Binding in Cerebellar Degenerations Studied with Positron Emission Tomography
}

\author{
Sid Gilman, MD, ${ }^{*}$ Robert A. Koeppe, PhD, $\dagger$ Larry Junck, MD, ${ }^{*}$ Karen J. Kluin, MS, $\stackrel{*}{\dagger}$ \\ Mary Lohman, BA, ${ }^{*}$ and Roy T. St. Laurent, PhD $\$$
}

\begin{abstract}
We used positron emission tomography with $\left[{ }^{11} \mathrm{C}\right]$ flumazenil to study gamma-aminobutyric acid type A/benzodiazepine receptor binding quantitatively in the cerebral hemispheres, basal ganglia, thalamus, cerebellum, and brainstem of 72 subjects, including 14 with multiple system atrophy of the ataxic (olivopontocerebellar atrophy) type, 5 with multiple system atrophy of the extrapyramidal/autonomic (Shy-Drager syndrome) type, 18 with sporadic olivopontocerebellar atrophy, 15 with dominantly inherited olivopontocerebellar atrophy, and 20 normal control subjects with similar age and sex distributions. In comparison with data obtained from the normal control subjects, we found significantly decreased ligand influx in the cerebellum and brainstem of multiple system atrophy patients of the olivopontocerebellar atrophy type and in patients with sporadic olivopontocerebellar atrophy, but not in patients with multiple system atrophy of the Shy-Drager syndrome type. Despite these differences in ligand influx, benzodiazepine binding was largely preserved in the cerebral hemispheres, basal ganglia, thalamus, cerebellum, and brainstem in patients with multiple system atrophy of both types as well as those with sporadic or dominantly inherited olivopontocerebellar atrophy as compared with normal control subjects. The finding of relative preservation of benzodiazepine receptors indicates that these sites are available for pharmacological therapy in these disorders.
\end{abstract}

Gilman S, Koeppe RA, Junck L, Kluin KJ, Lohman M, St. Laurent RT. Benzodiazepine receptor binding in cerebellar degenerations studied with positron emission tomography. Ann Neurol 1995;38:176-185

Gamma-aminobutyric acid (GABA) is an inhibitory neurotransmitter located abundantly in the central nervous system (CNS) $[1,2]$. Most GABAergic neurons are interneurons, but some such as Purkinje cells and striatonigral, nigrothalamic, and nigrotectal neurons are principal output neurons. Two types of receptor sites mediate GABAergic neurotransmission, $\mathrm{GABA}_{\mathrm{A}}$ and $G_{A B A}$. The action of the $G A B A_{A}$ receptor is the opening of chloride channels $[3,4]$. Low- and highaffinity $G A B A_{A}$ receptor binding sites exist in the mammalian central nervous system [5-9]. Low-affinity $\mathrm{GABA}_{\mathrm{A}}$ receptors contain at least three binding sites for pharmacologically specific agents, including benzodiazepines (BDZ), barbiturates, and convulsants [2, 3 , 10, 11]. These sites are functionally coupled to one another, residing on the oligomeric protein complex that regulates chloride ion permeability [2]. The recent development of a BDZ ligand, $\left[{ }^{11} \mathrm{C}\right]$ flumazenil (FMZ), that can be used with positron emission tomography (PET) makes it possible to measure the density of $\mathrm{GABA}_{\mathrm{A}} / \mathrm{BDZ}$ receptors in the central nervous system of patients with disorders that affect neurons containing these receptors $[12-14]$.
Multiple system atrophy (MSA) is a progressive neurodegenerative disease presenting with combinations of extrapyramidal, pyramidal, cerebellar, and autonomic symptoms and signs [15-18]. Some patients with MSA have extrapyramidal and autonomic symptoms (Shy-Drager syndrome [SDS]). Others have only extrapyramidal symptoms unresponsive to levodopa throughout their course (striatonigral degeneration [SND]), but in most of these patients, neuropathological examination demonstrates degenerative changes in the cerebellum and brainstem [19]. Another pattern is initial extrapyramidal symptoms with the later development of cerebellar symptoms, with or without autonomic symptoms [15]. Other patients first manifest cerebellar symptoms at which time they might be thought clinically to have sporadic olivopontocerebellar atrophy (sOPCA), but later they develop autonomic or extrapyramidal symptoms and then fall into the diagnostic category of MSA. The neuropathological changes in MSA include those seen in SND [20-23], SDS [17, 24-27], and OPCA [17-19]. Neuronal loss and gliosis occur in the basal ganglia (putamen and globus pallidus), brainstem and cerebellum (substantial nigra, locus ce-
From the *Department of Neurology; †Division of Nuclear Medicine, Department of Internal Medicine; $\$$ Division of Speech Pathology, Department of Physical Medicine and Rehabilitation; and $\$$ Department of Biostatistics, University of Michigan, Ann Arbor, MI, and Department of Mathematics, Northern Arizona University, Flagstaff, AZ 86011-5717.
Received Aug 8, 1994, and in revised form Dec 13, 1994, and Feb 16 and Mar 27, 1995. Accepted for publication Mar 30, 1995.

Address correspondence to Dr Gilman, Department of Neurology, University of Michigan Medical Center, 1500 E. Medical Center Drive, Ann Arbor, MI 48109-0316. 
ruleus, dorsal vagal nuclei, vestibular nuclei, inferior olives, pontine nuclei, cerebellar Purkinje cells), and spinal cord (pyramidal tracts, intermediolateral columns and Onuf's nuclei) $[15,18,19]$. Many of the structures affected contain neurons with $\mathrm{GABA}_{\mathrm{A}} / \mathrm{BDZ}$ receptors. Recently, oligodendroglial [28-32] and neuronal [33-35] intracytoplasmic and intranuclear inclusions have been found in MSA.

OPCA is a progressive neurological disorder characterized by degeneration of neurons in the inferior olives, pons, and cerebellum occurring both sporadically and with hereditary transmission [36-40]. When hereditary, the disease is usually expressed as an autosomal dominant trait, but at times as an autosomal recessive trait $[39,40]$. A locus on chromosome 6 was identified in some families with dominantly inherited OPCA [41], and loci also were found on chromosomes 12,14 , and 16 in orher families. Most patients become symptomatic in middle age with progressive ataxia of gair and speech, disturbances of extraocular movements, and difficulty with limb coordination. The neuropathological changes include loss of inferior olivary neurons and their climbing fiber projections to the cerebellum, and loss of pontine neurons and their mossy fiber connections in the cerebellum [18, 42-44]. Purkinje cells are severely reduced but not completely lost, and granule cells are reduced. Golgi cells are probably unaffected, and basket cells are relatively preserved. Dentate neurons also are preserved. Many of the neurons affected in OPCA contain $\mathrm{GABA}_{\mathrm{A}} / \mathrm{BDZ}$ receptors. In some patients, OPCA evolves clinically into MSA and patients show degenerative changes within the basal ganglia and spinal cord [15-17]. Most patients who present initially with OPCA and later develop clinical manifestations of MSA have a sporadic and not a hereditary form of the disorder [16]. It is unclear whether all patients with SOPCA progress to develop MSA. In the present study, we separated patients into OPCA and MSA groups based on clinical criteria provided in the Materials and Methods section.

The present study was initiated to examine BDZ receptors in the cerebellum of patients with MSA, sOPCA, and dominantly inherited OPCA (dOPCA) in comparison with normal control subjects with similar age and sex distributions, to determine whether these receptors are preserved in these disorders. The patients with MSA were further subdivided into those with the ataxic (olivopontocerebellar atrophy) type $\left(\mathrm{MSA}_{\mathrm{OPCA}}\right)$ and those with the extrapyramidal/autonomic (SDS) type $\left(\mathrm{MSA}_{\mathrm{SDS}}\right)$. We were interested in $\mathrm{BDZ}$ receptors because differences in $\mathrm{BDZ}$ receptor density may (1) give insights into the pathogenesis of these disorders, (2) suggest new approaches to diagnosis, and (3) indicate whether $\mathrm{GABA}_{\mathrm{A}} / \mathrm{BDZ}$ receptors are available for potential pharmacological interventions in these disorders.

\section{Materials and Methods \\ Patient Groups and Normal Subjects}

The studies were approved by the Institutional Review Board, and informed consent was obtained from each subject. Five groups of subjects were studied: (1) MSA $\mathrm{OPCA}_{\text {, }}$ (2) $\mathrm{MSA}_{\mathrm{SDS}}$, (3) SOPCA, and (4) dOPCA patients, and (5) normal control subjects (Table 1 ). We studied 72 subjects ( 52 patients and 20 normal control subjects), mean age 56 \pm 13 years ( \pm standard deviation), including 36 women ( 9 normal control subjects and 27 patients), mean age $54 \pm 14$ years, and 36 men (11 normal control subjects and 25 patients), mean age $58 \pm 12$ years (see Table 1). Significant differences in age were found among the five diagnosis groups $(p<0.0005)$ with a single factor analysis of variance (ANOVA). Three patients, 2 with sOPCA and 1 with IOPCA, were included from our previous positron emission tomography (PET) studies of glucose metabolic rate with $\left[{ }^{18} \mathrm{~F}\right]$ fluorodeoxyglucose (FDG) [45-47]. One of the 2 patients with sOPCA in the previous communications developed extrapyramidal and autonomic symptoms and signs subsequent to the initial study and is now included in the $\mathrm{MSA}_{\mathrm{OPCA}}$ group. In our analysis of the duration of symptoms, we report the median and range rather than the mean and standard deviation because the distributions are skewed. For the MSA $A_{\mathrm{OPCA}}$ group the duration ranged from 2 to 11 years with a median of 5 years; for the MSA $_{\mathrm{SDS}}$ group the range was 2 to 16 years with a median of 7 years; for the sOPCA group, the range was 1 to 20 years with a median of 4.5 years; and for the dOPCA group, the range was 2 to 20 years with a median of 5 years. The normal control subjects had no history of neurological disorder and no abnormalities on general physical and neurological examinations. At the time of the PET study, none of the patients or normal control

Table 1. Numbers, Ages (mean \pm standard deviation), and Sex Distribution of the Subjects Studied

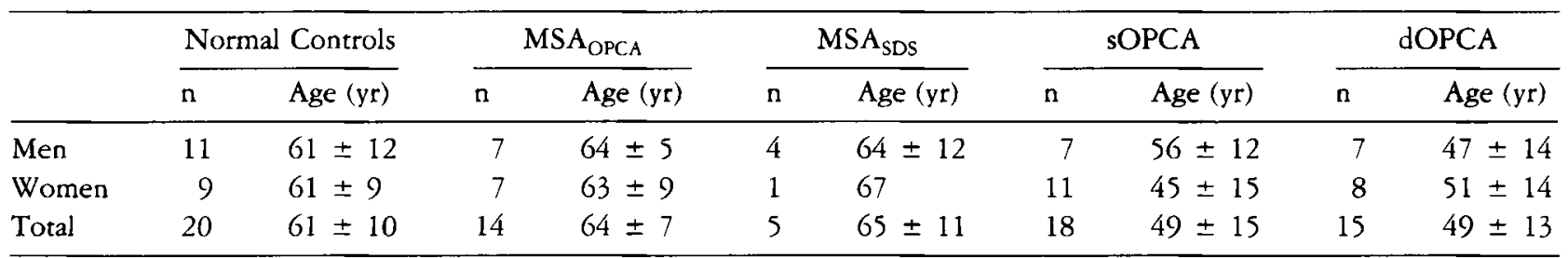

MSA = multiple system atrophy; $\mathrm{MSA}_{\mathrm{OPCA}}=\mathrm{MSA}$ group with cerebellar and autonomic or cerebellar, extrapyramidal, and autonomic signs; $M S A_{S D S}=$ MSA group with extrapyramidal and autonomic signs only; sOPCA = sporadic olivopontocerebellar atrophy; dOPCA = dominantly inherited olivopontocerebellar atrophy. 
subjects were taking medications that are known to influence $B D Z$ receptors. Five of the MSA patients had been treated with $\mathrm{BDZ}$ (diazepam, chlordiazepoxide, or clonazepam), but these medications had been discontinued for 6 weeks before the PET study in 2 patients and for 1 to 3 years in the other 3. Two of the dOPCA patients had been treated with $B D Z$ (chlordiazepoxide or clonazepam), and these medications were discontinued 6 weeks and 1 year prior to PET study. Three of the patients with SOPCA had been treated with BDZ (clonazepam), and these medications had been discontinued for 6 weeks to 2 years prior to PET study. All 5 of the $\mathrm{MSA}_{\mathrm{SDS}}$ patients were taking medications for parkinsonism (carbidopa/levodopa $25 / 100 \mathrm{mg}$ or $25 / 250 \mathrm{mg}$, three or four times daily).

Of the 19 patients with MSA, utilizing the criteria of Quinn [15], we made the diagnosis of probable MSA in 15 and definite MSA (autopsy verified) in 4. The diagnosis of probable MSA was based on demonstration of clinical signs of at least two of the following: (1) extrapyramidal disorder, (2) autonomic failure, and (3) cerebellar dysfunction. For the demonstration of extrapyramidal disorder, we required at least two of the following signs: akinesia, rigidity, tremor, and hypokinetic speech. Tremor was accepted as an extrapyramidal sign only if it was present at rest and involved distal parts of the limbs. With these criteria, we found that 16 of the 19 patients had extrapyramidal disorders. In 12 of the 16 , we also assessed the degree of responsiveness to levodopa with clinical neurological evaluations before and after treatment with carbidopa/levodopa at a dose of $25 / 250 \mathrm{mg}$, gradually increased from one to four tablets daily. Patients were judged to be unresponsive or mildly, moderately, or markedly responsive. Nine of the 12 tested were unresponsive, 1 was mildly responsive, 1 was moderately responsive, and 1 was markedly responsive. The remaining 4 patients could not be tested because they were referred to us from distant locations. The demonstration of changes in the putamen in magnetic resonance (MR) scans $[48,49\}$ provided helpful information for the diagnosis of MSA but was not required since these changes are not consistently present in MSA [50].

For the demonstration of cerebellar dysfunction, we required at least two signs, including limb and/or gait ataxia along with ocular dysmetria or ataxic dysarthria. We found that 14 of the 19 MSA patients had cerebellar dysfunction. For the demonstration of autonomic failure, we required postural hypotension, sexual impotence (in the males), or urinary incontinence without outflow obstruction. The criteria for postural hypotension were similar to those of McLeod and Tuck [51], including an orthostatic drop of $30 \mathrm{~mm} \mathrm{Hg}$ or more in systolic blood pressure and $20 \mathrm{~mm} \mathrm{Hg}$ or more in diastolic blood pressure with an increase in heart rate of no more than 10 beats $/ \mathrm{min}$. Blood pressure and pulse were measured with the patient supine and again 2 minutes after the patient had assumed the standing position. In patients who could not tolerate standing for 2 minutes, these changes were accepted after the patient stood as long as tolerated. None of the patients were taking medications that induce postural hypotension except for carbidopa/levodopa, which was administered to $5 \mathrm{MSA}_{\mathrm{SDS}}$ patients. Eleven of the 19 patients with MSA had postural hypotension, 15 of the 19 had urinary incontinence, 9 of the 19 had both postural hypotension and urinary incontinence, and 10 of 11 male patients had sexual impotence. All 19 patients had at least one of the criteria for autonomic insufficiency. In summary, 11 of the 19 patients with MSA had extrapyramidal, cerebellar, and autonomic dysfunction; 5 had extrapyramidal and autonomic disturbances; and 3 had cerebellar and autonomic disorders. The 5 with extrapyramidal and autonomic disturbances constituted the MSA $A_{S D S}$ group; the other 14, in whom cerebellar dysfunction dominated, made up the MSA $_{\text {OPCA }}$ group.

The diagnosis of OPCA was based on a history of progressive deterioration in cerebellar function manifested by ataxia of gait and speech, usually accompanied by ataxia of ocular and limb movements, in the absence of a disorder of sensory function sufficient to cause ataxia, medications (e.g., phenytoin), toxins (e.g., alcohol), evidence of a neoplasm in the cerebellum or elsewhere, or evidence of multiple sclerosis or other diseases that can cause progressive cerebellar ataxia. The diagnosis was assisted by the demonstration of cerebellar and brainstem atrophy in computed tomography (CT) or MR scans, but this was not required since OPCA can occur in the absence of atrophy on anatomical imaging studies [45].

Each patient was evaluated with a complete history, physical examination, neurological examination, laboratory tests to exclude other diseases, and MR imaging to exclude demyelinative disease and structural abnormalities. Speech was evaluated as described previously [47]. Laboratory tests included a complete blood cell count; serum profiles of hepatic and renal function; brainstem auditory, visual, and somatosensory evoked potentials; serum levels of vitamins $E$ and $B_{12}$ and folic acid; and studies of thyroid function. In patients with symptoms for less than 3 years, a search was made for an occult malignancy, including breast and pelvic examinations in women, prostate examination in men, measurement of acid phosphatase and prostate-specific antigen levels, stool guaiac tests for occult blood, and chest x-ray films. In addition, anti-Purkinje cell antibodies were sought in blood samples for patients with ataxia of less than 3 years' duration. All patients were evaluated with MR imaging to determine the extent of volume loss of the structures under study. The diagnosis of SOPCA was made if there was no family history of a similar illness after a detailed history had been taken with construction of a family tree. The diagnosis of dOPCA was based on a clear family history of a similar disorder in at least one parent. Patients with a family history suggesting autosomal recessive inheritance were excluded from this study.

The neurological examinations were conducted by a neurologist (S. G.) blinded to the PET data. Speech was evaluated by a speech-language parhologist (K. J. K.) also blinded to the results of the PET studies.

\section{Positron Emission Tomograpby Studies}

PET studies were conducted similarly for all subjects. The subjects were fasted for 4 hours before scanning. A catheter was placed in a radial artery for blood sampling. The subjects lay quietly on a table, eyes open, ears unoccluded, and alert but not speaking. Scans were performed after intravenous injection of $\left[{ }^{11} \mathrm{C}\right] \mathrm{FMZ}$ in patients with MSA, SOPCA, dOPCA, and normal control subjects.

The subjects were imaged with a Siemens/CTI 931/08-12 scanner, which has an intrinsic in-plane resolution of $5.5-\mathrm{mm}$ full-width at half-maximum (FWHM) at the center of the 
field of view and an axial resolution of 7.0-mm FWHM. The reconstructed resolution is $9.0-\mathrm{mm}$ FWHM at the center of the field of view. Fifteen planes with a 6.75 -mm center-tocenter separation were imaged simultaneously. Attenuation correction was calculated by the standard ellipse method. The PET studies were analyzed with a compartmental model and parameter estimation technique that provides pixel-by-pixel determinations of each measurement, thus creating "functional" images [13].

We labeled FMZ with ${ }^{11} \mathrm{C}$ at high specific radioactivity using a methylation process [12] and administered a dose of $22 \pm 2 \mathrm{mCi}$. Blood samples were taken as rapidly as possible during the first 2 minutes after tracer injection and then at progressively longer intervals throughout the remainder of the study. We took a total of 25 to 30 samples per scan. The samples were centrifuged, and the plasma radioisotope concentrations were measured in an $\mathrm{Nal}$ well counter. Plasma levels of radiolabeled metabolites of $\left[{ }^{11} \mathrm{C}\right] \mathrm{FMZ}$ were determined by a rapid Sep-Pak $C_{18}$ cartridge chromatographic technique [14] in the samples taken at 1 minute and 2 minutes, and every sample from 3 minutes until the end of the scan. Dynamic PET scanning was performed for 60 minutes beginning immediately after injection. Radioactive fiducials with $5 \mu \mathrm{Ci}$ of $\left[{ }^{11} \mathrm{C}\right] \mathrm{FMZ}$ were placed on each subject's scalp. Computer routines automatically determined the locations of these fiducials and used this information to correct for patient motion. The studies with $\left[{ }^{11} \mathrm{C}\right] \mathrm{FMZ}$ were performed to measure ligand influx $\left(K_{1}\right)$, which is highly correlated with flow since the single-pass extraction fraction is greater than $50 \%$ [13]. The studies were also performed to measure ligand distribution volume (DV), which is linearly related to the density of available receptor sites divided by the ligand dissociation constant $\left(B_{\max } ' / K_{\mathrm{D}}\right)$. The methods of using $\left[{ }^{11} \mathrm{C}\right] \mathrm{FMZ}$ for $\mathrm{BDZ}$ receptor binding, including the assumptions and limitations and the performance of the agent, are published [13, 14, 52].

A data analyst (M. L.) extracted volume of interest (VOI) information from the PET studies, and these data were shared among the investigators only after completion of measurements from all studies. The process of data extraction is almost completely automated, requiring essentially no judgments by the data analyst. For VOI placement we utilized templates developed previously $[45-47,53]$. The PET studies were displayed as two image sets (one each for FMZ $K_{1}$ and DV). VOIs were identified in the identical areas from each matched set. VOI data were acquired from the cerebral cortex, caudate nucleus, putamen, thalamus, cerebellar hemispheres, cerebellar vermis, and brainstem. Although study of the cerebellar nuclei would be of interest, the binding in this structure with FMZ was not sufficient to allow accurate measurement. PET images were viewed in the transverse, sagittal, and coronal planes. Each pixel measures $1.875 \times$ $1.875 \mathrm{~mm}$. Data from the cerebral cortex were obtained in transverse images by measuring $K_{1}$ and $\mathrm{DV}$ in the cortical ribbon from four or five consecutive planes of 15 , beginning with the plane containing the most superior portion of the cingulate gyrus and continuing ventrally to the plane containing the lowest portion of the thalamus. This was accomplished with an algorithm that detects the outer edge of the cortical rim from an image that has been passed through a contrast-enhancing filter. The algorithm then identified a cortical band on the original image that extends inward from this edge until the DV drops below the value on the outer edge of the rim or the band reaches a width of $15 \mathrm{~mm}$. For analysis of the basal ganglia, data were obtained from a 0.9 $\mathrm{cm}^{2}$ square over the caudate nucleus of each side from 2 of the 15 levels, a $1.9-\mathrm{cm}^{2}$ parallelogram over each putamen from 2 of the 15 levels, and a $1.3-\mathrm{cm}^{2}$ parallelogram over the thalamus of each side from 2 of the 15 levels. For the cerebellum, data were collected from two slices utilizing images obtained in the transverse plane with a $2.6-\mathrm{cm}^{2}$ polygon over each cerebellar hemisphere. Data were collected from the cerebellar vermis posterior to the fourth ventricle utilizing a $6 \times 10 \times 7$-voxel parallelepiped on a midsagittal slice. Data were collected in the midsagittal plane from the pons with a $1.3 \times 1.5 \times 1.1-\mathrm{cm}$ right parallelepiped. Each VOI was centered over a local peak in $K_{1}$.

\section{Data Analysis}

The data were analyzed in several stages. In the first stage, not presented here, plots of the data were used to assess gross trends and relationships between variables. Second, a multivariate repeated measures analysis of covariance (MANCOVA) model was fitted to the data incorporating region as the repeated (within-subject) measures variable, diagnosis group, sex, and age, and all of the interactions among these variables to determine whether differences exist between brain regions. In the multivariate analysis, the dependent variable was regional brain $K_{1}$ or DV (either absolute or normalized). Measurements on seven (or six) brain regions constituted the repeated measurements on each patient for absolute (or normalized) data. The primary independent variable in the analysis was the categorical variable diagnosis group (MSA OPCA $_{1}$, MSA $_{\text {SDS }}$, SOPCA, dOPCA, or normal control). The factor sex and the continuous covariate age also were included in the model. All possible interactions between diagnosis group, sex, and age were considered. Age was standardized by subtracting the mean age across all subjects.

Depending on the outcome of the analysis in the second stage, a univariate ANOVA or ANCOVA model was developed to examine each region separately. The factors incorporated into the univariate model were those identified in the second stage as having an overall effect on $K_{1}$ or DV across regions or having a differential effect on $K_{1}$ or $\mathrm{DV}$ between regions. In the last stage, comparisons of individual diagnosis group means were made where appropriate. For all pairwise differences a Bonferroni multiple comparison adjustment [54] was used. With five diagnosis groups under comparison, examination of all comparisons among the age-adjusted group means provided 10 comparisons, which was the Bonferroni factor used throughout. Data were analyzed with the SAS statistical package (SAS Institute, Cary, NC).

\section{Results \\ Flumazenil Ligand Influx}

We studied FMZ influx $\left(K_{1}\right)$ (Fig 1) using both absolute values (Table 2 ) and values normalized to the whole brain cerebral cortex (Table 3). In the multivariate repeated MANCOVA of absolute $K_{1}$, we found no evidence for differential effects of age by region or 


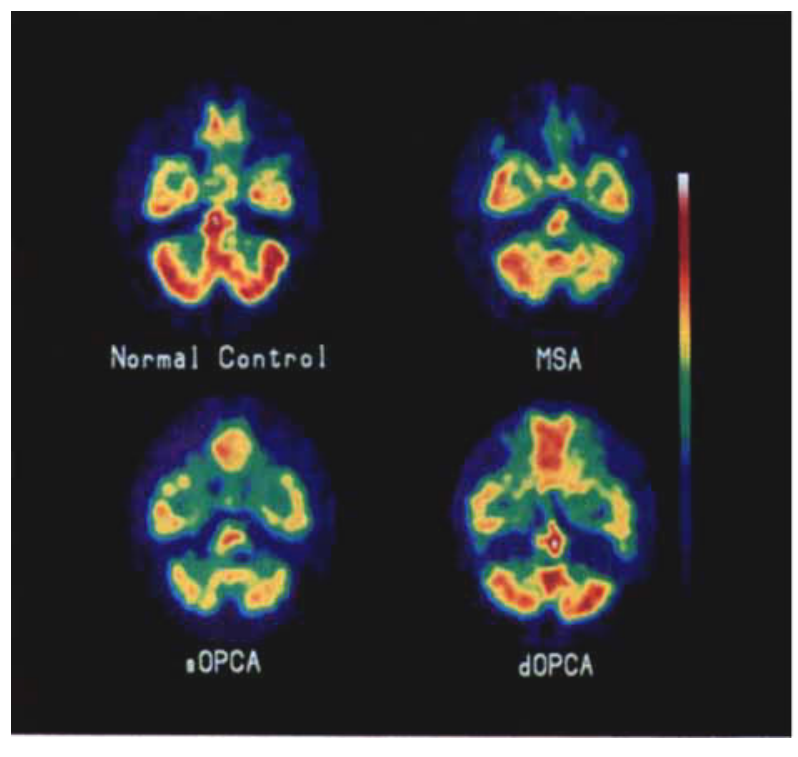

Fig 1. Positron emission tomograpby scans with $\left({ }^{11} \mathrm{C}\right)$ flumazenil sbowing ligand influx $\left(\mathrm{K}_{1}\right)$ in a male normal control subject aged 53 years (left upper image), a male patient aged 71 years with multiple system atropby (MSA, right upper image) of the ataxic (olivopontocerebellar atrophy) type, a male patient aged 63 years with sporadic olivopontocerebellar atropby (sOPCA, left lower image), and a male patient aged 60 years with dominantly inberited olivopontocerebellar atrophy (dOPCA, right lower image). All scans show borizontal sertions at the level of the cerebellum and the base of the temporal and frontal lobes. The scans show decreased influx in the cerebellum and brainstem in MSA and SOPCA and in the cerebellum in OOPCA in comparison with the normal subject.

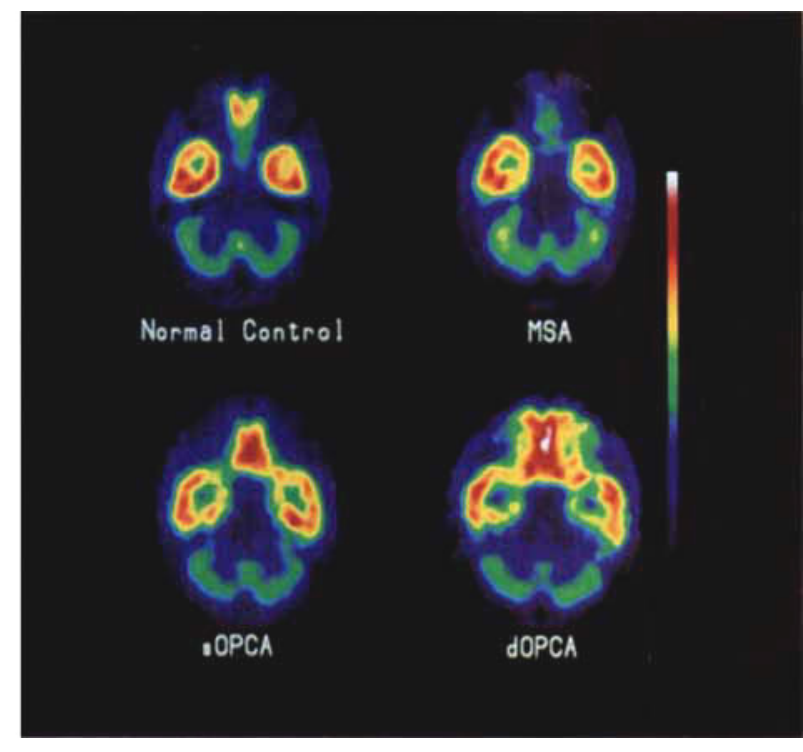

Fig 2. Positron emission tomography scans with $\left({ }^{11} \mathrm{C}\right)$ flumazenil showing ligand distribution volume (DV) in the same subjects shown in Figure 1: a male normal control subject aged 53 years (left upper image), a male patient aged 71 years with multiple system atropby (MSA, right upper image) of the ataxic (olivopontocerebellar atrophy) type. a male patient aged 63 years with sporadic olivopontocerebellar atrophy (sOPCA, left lower image), and a male patient aged 60 years with dominantly inberited olivopontocerebellar atropby (dOPCA, right lower image). All scans show borizontal sections at the level of the cerebellum and the base of the temporal and frontal lobes. The color bar indicates $\left(^{I} \mathrm{C}\right.$ flumazenil $D V$ in milliliters per gram. The scans show essentially equal $D V$ in the patients as compared with the normal subject.

Table 2. Flumazenil Influx $\left(K_{1}\right)$. Absolute Levels (in $\mathrm{ml} / \mathrm{gm} / \mathrm{min}$, mean \pm standard error) ${ }^{\mathrm{a}}$

\begin{tabular}{llllll}
\hline & $\begin{array}{l}\text { Normal } \\
\text { Controls } \\
(\mathbf{n}=20)\end{array}$ & $\begin{array}{l}\mathrm{MSA}_{\text {OPCA }} \\
(\mathbf{n}=14)\end{array}$ & $\begin{array}{l}\mathrm{MSA}_{\text {SOS }} \\
(\mathbf{n}=5)\end{array}$ & $\begin{array}{l}\text { sOPCA } \\
(\mathbf{n}=18)\end{array}$ & $\begin{array}{l}\text { dOPCA } \\
(\mathbf{n}=15)\end{array}$ \\
\hline Cerebral cortex & $0.29 \pm 0.01$ & $0.31 \pm 0.02$ & $0.30 \pm 0.03$ & $0.29 \pm 0.01$ & $0.32 \pm 0.02$ \\
Caudate nuclei & $0.36 \pm 0.02$ & $0.37 \pm 0.03$ & $0.37 \pm 0.04$ & $0.34 \pm 0.02$ & $0.40 \pm 0.02$ \\
Putamen & $0.38 \pm 0.02$ & $0.39 \pm 0.03$ & $0.42 \pm 0.04$ & $0.37 \pm 0.02$ & $0.42 \pm 0.02$ \\
Thalamus & $0.43 \pm 0.02$ & $0.46 \pm 0.04$ & $0.47 \pm 0.05$ & $0.41 \pm 0.02$ & $0.47 \pm 0.03$ \\
Cerebellar hemispheres & $0.37 \pm 0.02$ & $0.25 \pm 0.03^{\mathrm{b}}$ & $0.36 \pm 0.05$ & $0.25 \pm 0.02^{\mathrm{c}}$ & $0.34 \pm 0.02$ \\
Cerebellar vermis & $0.35 \pm 0.02$ & $0.27 \pm 0.02$ & $0.38 \pm 0.04^{\mathrm{d}}$ & $0.25 \pm 0.02^{\mathrm{d} . e}$ & $0.32 \pm 0.02$ \\
Brainstem & $0.33 \pm 0.02$ & $0.24 \pm 0.03$ & $0.37 \pm 0.04$ & $0.25 \pm 0.02^{\mathrm{b}}$ & $0.31 \pm 0.02$ \\
\hline
\end{tabular}

${ }^{2}$ Abbreviations are as in Table 1.

significantly different from normal controls at $p<0.05$.

'Significantly different from normal controls at $p<0.005$.

${ }^{\triangleleft}$ Significant difference between $\mathrm{MSA}_{\mathrm{SDS}}$ and SOPCA at $p<0.05$.

'Significantly different from normal controls at $p<0.001$. 


\begin{tabular}{llllll}
\hline Structure & $\begin{array}{l}\text { Normal } \\
\text { Controls } \\
(\mathrm{n}=20)\end{array}$ & $\begin{array}{l}\mathrm{MSA}_{\mathrm{OPCA}} \\
(\mathrm{n}=14)\end{array}$ & $\begin{array}{l}\mathrm{MSA}_{\mathrm{SDS}} \\
(\mathrm{n}=5)\end{array}$ & $\begin{array}{l}\text { sOPCA } \\
(\mathrm{n}=18)\end{array}$ & $\begin{array}{l}\text { dOPCA } \\
(\mathrm{n}=15)\end{array}$ \\
\hline Caudate nuclei & $1.24 \pm 0.03$ & $1.20 \pm 0.05$ & $1.23 \pm 0.07$ & $1.17 \pm 0.03$ & $1.23 \pm 0.03$ \\
Putamen & $1.33 \pm 0.02$ & $1.27 \pm 0.04$ & $1.40 \pm 0.06$ & $1.29 \pm 0.03$ & $1.31 \pm 0.03$ \\
Thalamus & $1.50 \pm 0.03$ & $1.49 \pm 0.06$ & $1.57 \pm 0.08$ & $1.42 \pm 0.04$ & $1.46 \pm 0.04$ \\
Cerebellar hemispheres & $1.27 \pm 0.05$ & $0.81 \pm 0.08^{\mathrm{b}}$ & $1.18 \pm 0.12$ & $0.88 \pm 0.05^{\mathrm{b}}$ & $1.05 \pm 0.06^{\mathrm{c}}$ \\
Cerebellar vermis & $1.22 \pm 0.04$ & $0.88 \pm 0.06^{\mathrm{d}, \mathrm{e}}$ & $1.25 \pm 0.09^{\mathrm{e}, \mathrm{f}}$ & $0.87 \pm 0.04^{\mathrm{b}, \mathrm{f}}$ & $0.99 \pm 0.05^{\mathrm{g}}$ \\
Brainstem & $1.13 \pm 0.04$ & $0.80 \pm 0.07^{\mathrm{e}, \mathrm{g}}$ & $1.23 \pm 0.11^{\mathrm{e}, \mathrm{h}}$ & $0.85 \pm 0.05^{\mathrm{b}, \mathrm{h}}$ & $0.98 \pm 0.05$ \\
\hline
\end{tabular}

${ }^{2}$ Abbreviations are as in Table 1.

${ }^{3}$ Significantly different from normal controls at $p<0.0001$.

'Significantly different from normal controls at $p<0.05$.

${ }^{\circ}$ Significantly different from normal controls at $p<0.001$.

'Significant difference between $\mathrm{MSA}_{\mathrm{OPCA}}$ and $\mathrm{MSA}_{\mathrm{SDS}}$ at $p<0.05$.

'Significant difference between MSA $A_{S D S}$ and SOPCA at $p<0.005$.

${ }^{B}$ Significantly different from normal controls at $p<0.005$.

${ }^{\mathrm{h}}$ Significant difference between MSA $\mathrm{SDS}_{\text {and }}$ SOPCA at $p<0.05$.

sex by region, nor evidence for differential effects of interactions among these factors and diagnosis by region. Strong evidence was obtained for differences in regions $(p<0.0001)$ and for differential effects of diagnosis by region. Further (univariate) analysis of individual regions, adjusted for age and age by diagnosis interactions, revealed highly significant differences between the five diagnostic groups in the cerebellar hemispheres $(p=0.001)$ and cerebellar vermis $(p=$ $0.001)$. A significant difference was found in brainstem $(p=0.005)$ as well. No differences among diagnostic groups were found in the cerebral cortex, caudate nucleus, putamen, or thalamus ( $p>0.10$ ).

As indicated in Table 2, pairwise comparisons of age-adjusted means for the groups found to differ as described above (cerebellar hemispheres, cerebellar vermis, and brainstem), corrected with a Bonferroni adjustment, revealed significantly decreased absolute $K_{1}$ in the cerebellar hemispheres $(p<0.005)$, cerebellar vermis $(p<0.001)$, and brainstem $(p<0.05)$ in sOPCA patients compared to normal control subjects. The analysis also showed significantly decreased absolute $K_{1}$ in the cerebellar hemispheres $(p<0.05)$ in $\mathrm{MSA}_{\mathrm{OPCA}}$ patients compared to normal control subjects and in the cerebellar vermis $(p<0.05)$ in SOPCA compared to $\mathrm{MSA}_{\mathrm{SDS}}$ patients.

Analysis of $K_{1}$ normalized to cerebral cortex revealed no evidence for differential effects of sex or age by region, nor evidence for differential effects of interactions between sex and diagnosis or age by region. Strong evidence was found for differences in regions $(p<0.0001)$ and differential effects of diagnosis by region $(p<0.003)$. Further analysis of individual regions, adjusted for age and age by diagnosis interactions, demonstrated highly significant differences between the five diagnostic groups in the cerebellar hemispheres $(p<0.00001)$, cerebellar vermis $(p<$
$0.00001)$, and brainstem $(p=0.00001)$. No other regions exhibited significant differences in diagnostic groups $(p>0.10)$.

Individual Bonferroni-adjusted comparisons of ageadjusted mean normalized $K_{1}$ values (see Table 3) revealed a significant decrease of influx in the cerebellar hemispheres $(p<0.0001)$, cerebellar vermis $(p<$ $0.001)$, and brainstem $(p<0.005)$ in $\mathrm{MSA}_{\mathrm{OPCA}}$ patients in comparison to normal subjects. A significant decrease of $K_{1}$ was found in these same three regions (all $p<0.0001$ ) in SOPCA patients in comparison to normal control subjects. A significant decrease of $K_{1}$ was found only in the cerebellar hemispheres $(p<$ $0.05)$ and cerebellar vermis $(p<0.05)$ in DOPCA patients in comparison to normal control subjects. A significant decrease of $K_{1}$ was found in the cerebellar vermis and brainstem $(p<0.05)$ in $\mathrm{MSA}_{\mathrm{OPCA}}$ in comparison to $\mathrm{MSA}_{\mathrm{SDS}}$ patients, and a significant decrease of $K_{1}$ was found in the cerebellar vermis $(p<0.005)$ and brainstem $(p<0.05)$ in SOPCA in comparison to $\mathrm{MSA}_{\mathrm{SDS}}$ patients.

\section{Flumazenil Ligand Distribution Volume}

In the multivariate repeated MANCOVA of absolute DV, we found no evidence of differences among the diagnostic groups (Fig 2). We found neither an average diagnosis effect nor a differential diagnosis effect by region. Univariate ANCOVA adjusted for age and age by diagnosis interactions supported this finding (Table 4).

Analysis of DV values normalized to the cerebral cortex provided evidence for a differential effect of diagnosis by region as well as age by diagnosis by region interaction. No average effect of diagnosis across all regions was apparent. Subsequent univariate analyses of each region disclosed differences between diagnostic groups only in the cerebellar vermis $(p=0.01)$. 
Table 4. Flumazenil Distribution Volume, Absolute Levels (in $\mathrm{ml} / \mathrm{gm}$, mean \pm standard error) ${ }^{a}$

\begin{tabular}{llllll}
\hline & $\begin{array}{l}\text { Normal } \\
\text { Controls } \\
(\mathbf{n}=20)\end{array}$ & $\begin{array}{l}\text { MSA } \\
(\mathbf{n}=\mathbf{1 4})\end{array}$ & $\begin{array}{l}\text { MSA } \\
(\mathbf{n}=5)\end{array}$ & $\begin{array}{l}\text { sOPCA } \\
(\mathbf{n}=18)\end{array}$ & $\begin{array}{l}\text { dOPCA } \\
(\mathbf{n}=15)\end{array}$ \\
\hline Cerebral cortex & $5.23 \pm 0.20$ & $5.45 \pm 0.33$ & $5.70 \pm 0.49$ & $5.58 \pm 0.22$ & $5.52 \pm 0.24$ \\
Caudate nuclei & $3.07 \pm 0.18$ & $3.23 \pm 0.30$ & $3.07 \pm 0.45$ & $3.25 \pm 0.20$ & $3.10 \pm 0.22$ \\
Putamen & $3.51 \pm 0.15$ & $3.91 \pm 0.25$ & $3.69 \pm 0.37$ & $3.75 \pm 0.16$ & $3.61 \pm 0.18$ \\
Thalamus & $3.59 \pm 0.14$ & $3.70 \pm 0.23$ & $3.74 \pm 0.34$ & $3.64 \pm 0.15$ & $3.68 \pm 0.17$ \\
Cerebellar hemispheres & $4.06 \pm 0.24$ & $4.74 \pm 0.38$ & $4.39 \pm 0.58$ & $4.29 \pm 0.26$ & $4.26 \pm 0.28$ \\
Cerebellar vermis & $3.58 \pm 0.17$ & $3.67 \pm 0.28$ & $3.84 \pm 0.43$ & $3.17 \pm 0.19$ & $3.39 \pm 0.21$ \\
Brainstem & $1.14 \pm 0.05$ & $1.12 \pm 0.08$ & $1.27 \pm 0.13$ & $1.12 \pm 0.06$ & $1.14 \pm 0.06$ \\
\hline
\end{tabular}

${ }^{2}$ No significant differences were found berween regions. Abbreviations are as in Table 1 .

Bonferroni-adjusted pairwise comparisons of ageadjusted means for each diagnostic group revealed significantly decreased DV in the cerebellar vermis $(p<$ $0.01)$ in sOPCA patients relative to normal control subjects (Table 5).

A power analysis was undertaken to determine whether the normal DV in all patient groups within all regions (except for the cerebellar vermis in SOPCA patients) was due to the limited number of subjects available. Using the estimated standard deviations and age-adjusted normal control means from these data, we examined the probability of detecting a $20 \%$ decrease in FMZ DV relative to normal control values for each region and diagnosis group for a test at $\alpha=$ 0.05 . For absolute data obtained from the cerebellar vermis, the power was 0.85 in SOPCA patients relative to normal control subjects, 0.81 for dOPCA patients, 0.80 for $\mathrm{MSA}_{\mathrm{OPCA}}$ patients, and 0.48 for $\mathrm{MSA}_{\mathrm{SDS}}$ patients. The power for the other regions in SOPCA patients ranged from a minimum of 0.69 for cerebral cortex to a maximum of 0.97 for caudate nucleus. For other diagnostic groups, the power ranged from 0.65 to 0.95 for DOPCA, 0.63 to 0.94 for $\mathrm{MSA}_{\mathrm{OPCA}}$, and 0.34 to 0.68 for $M S A_{S D S}$. For normalized data obtained from the cerebellar vermis, the power was 0.98 for
sOPCA, dOPCA, and MSA $A_{\mathrm{OPCA}}$ patients, and 0.78 for $M S A_{\text {SDs }}$ patients. The caudate nucleus was the region with the lowest power: 0.91 for sOPCA, 0.88 for dOPCA, 0.86 for $\mathrm{MSA}_{\mathrm{OPCA}}$, and 0.55 for $\mathrm{MSA}_{\mathrm{SDS}} \mathrm{pa-}$ tients. Thus, the lack of statistically significant differences in DV for most regions in most patient groups cannot be attributed to low power.

\section{Discussion}

This study demonstrated significantly decreased absolute levels of FMZ influx $\left(K_{1}\right)$ in the cerebellar hemispheres of patients with $\mathrm{MSA}_{\mathrm{OPCA}}$ as compared with normal control subjects; in the cerebellar hemispheres, cerebellar vermis, and brainstem of patients with sOPCA in comparison with normal control subjects; and in the cerebellar vermis of patients with sOPCA in comparison with $\mathrm{MSA}_{\mathrm{SDs}}$ patients. FMZ influx normalized to the cerebral cortex was significantly decreased in the cerebellar hemispheres and cerebellar vermis of patients with MSA ${ }_{\mathrm{OPCA}}$, SOPCA, or DOPCA, but not in patients with $M S A_{\text {SDS }}$ as compared with normal control subjects. FMZ influx normalized to the cerebral cortex was also significantly decreased in the brainstem of patients with $M S A_{\mathrm{OPCA}}$ or sOPCA but not in patients with $M S A_{S D S}$ or dOPCA. Since FMZ

Table 5. Flumazenil Distribution Volume, Normalized to the Cerebral Cortex (mean \pm standard error) ${ }^{\mathrm{a}}$

\begin{tabular}{llllll}
\hline & $\begin{array}{l}\text { Normal } \\
\text { Controls } \\
(\mathbf{n}=20)\end{array}$ & $\begin{array}{l}\text { MSA } \\
(\mathbf{n}=14)\end{array}$ & $\begin{array}{l}\text { MSA } \\
(\mathbf{n}=5)\end{array}$ & $\begin{array}{l}\text { sOPCA } \\
(\mathrm{n}=18)\end{array}$ & $\begin{array}{l}\text { dOPCA } \\
(\mathbf{n}=17)\end{array}$ \\
\hline Caudate nuclei & $0.59 \pm 0.03$ & $0.59 \pm 0.04$ & $0.56 \pm 0.06$ & $0.57 \pm 0.03$ & $0.56 \pm 0.03$ \\
Putamen & $0.67 \pm 0.02$ & $0.72 \pm 0.03$ & $0.66 \pm 0.04$ & $0.67 \pm 0.02$ & $0.65 \pm 0.02$ \\
Thalamus & $0.69 \pm 0.02$ & $0.68 \pm 0.03$ & $0.66 \pm 0.04$ & $0.66 \pm 0.02$ & $0.67 \pm 0.02$ \\
Cerebellar hemispheres & $0.78 \pm 0.03$ & $0.87 \pm 0.06$ & $0.79 \pm 0.08$ & $0.77 \pm 0.04$ & $0.76 \pm 0.04$ \\
Cerebellar vermis & $0.69 \pm 0.02$ & $0.68 \pm 0.04$ & $0.68 \pm 0.06$ & $0.57 \pm 0.03$ & $0.61 \pm 0.03$ \\
Brainstem & $0.22 \pm 0.01$ & $0.21 \pm 0.01$ & $0.22 \pm 0.02$ & $0.20 \pm 0.01$ & $0.21 \pm 0.01$ \\
\hline
\end{tabular}

${ }^{a}$ Abbreviations are as in Table 1.

${ }^{b}$ Significantly different from normal controls at $p<0.01$. 
influx depends strongly on cerebral blood flow (CBF), these findings suggest that $\mathrm{CBF}$ is decreased in the cerebellum of patients with the ataxic form of MSA $\left(\mathrm{MSA}_{\mathrm{OPCA}}\right)$ as well as both sporadic and dominantly inherited OPCA, but not in the extrapyramidal/autonomic form of MSA ( $\mathrm{MSA}_{\mathrm{SDS}}$ ). The decline of CBF values in these disorders probably results at least in part from partial volume effects related to tissue atrophy.

Despite the finding of diminished FMZ influx into the cerebellum in $\mathrm{MSA}_{\mathrm{OPCA}}$, SOPCA, and DOPCA and into the cerebellum and brainstem in $\mathrm{MSA}_{\mathrm{OPCA}}$ and sOPCA, DV was unchanged in all the structures studied, including the cerebellum and brainstem, except for a significant decrease in the cerebellar vermis in SOPCA with data normalized to the cerebral cortex. The decreased DV normalized to cerebral cortex in the cerebellar vermis in sOPCA resulted from the combination of a nonsignificant $11 \%$ decrease in absolute DV in the cerebellar vermis and a nonsignificant $7 \%$ increase in DV in the cerebral cortex. We cannot determine whether this finding is spurious, given that it results from the combination of a decrease in one region and an increase in another, or whether it reflects pathological involvement of neurons bearing BDZ receptors in the cerebellar vermis in SOPCA patients.

Subclinical striatal degeneration occurs frequently in OPCA, and thus it is interesting that striatal FMZ $K_{1}$ and DV values were normal in all patient groups. The substantial numbers of patients in the sOPCA, $\mathrm{dOPCA}$, and $\mathrm{MSA}_{\mathrm{OPCA}}$ groups and the power analysis lend credence to the findings in these patients, but with the small sample and relatively low power in the $\mathrm{MSA}_{\mathrm{SDS}}$ group, we do not view the results in this one group as definitive.

The cerebellar hemispheres, vermis, and brainstem are known to be decreased in volume in many patients with MSA or OPCA. Our methods do not permit determination of the extent to which atrophy affects our results. The method we used for analysis, that of placing a VOI of standard shape and size over the local peak of activity, is less sensitive to atrophy than are other methods. Nonetheless, it is possible that tissue atrophy partially accounts for our finding that some of the $K_{1}$ measurements in the cerebellar hemispheres, vermis, and brainstem are decreased in MSA $_{\mathrm{OPCA}}$, sOPCA, and DOPCA patients. Our measurements of DV generally showed no change, indicating little or no loss of BDZ receptors in these disorders. "Correcting" PET results to account for the effects of atrophy is an imprecise process, but had we attempted this, in all likelihood we would have found less prominent reductions in $K_{1}$. We cannot exclude the possibility that "correcting" the DV results would have yielded findings of increased DV per gram of tissue; the interpretation of such a finding would be that $G A B A_{A} / B D Z$ receptors are relatively spared in comparison to other tissue elements that are lost.

Shinotoh and colleagues [55] studied BDZ receptor binding with $\left[{ }^{11} \mathrm{C}\right] \mathrm{FMZ}$ and $\mathrm{PET}$ in a previous investigation of OPCA. This investigation revealed signifcantly increased binding (at the $p<0.01$ level) in the cerebellar cortex of 7 patients with sOPCA as compared with 6 normal control subjects. In the same study, BDZ receptor binding was decreased in the cerebellar cortex of 4 patients thought to have cerebellar cortical atrophy (CCA) in comparison with the normal control subjects, but this difference was not statistically significant. The findings in the present study did not confirm those of Shinotoh and colleagues [55]. Factors that might explain the disparity between the two studies include their separation of patients into OPCA and CCA groups, biological differences between subjects in the two studies, and the considerably larger number of patients in the present study.

Only two investigations utilizing postmortem brain tissue characterized the density of $\mathrm{GABA}_{\mathrm{A}} / \mathrm{BDZ}$ receptors in the cerebellum in MSA, and the findings in both studies are compatible with the results of the present investigation. In an autoradiographic study using $\left[{ }^{3} \mathrm{H}\right]$ flunitrazepam, Whitehouse and coworkers [56] found normal BDZ binding in the cerebellar cortex and increased binding in the dentate nuclei in 4 patients with cerebellar degeneration. Although all patients were labeled as having OPCA, 3 of the 4 met criteria for MSA of the ataxic type since their disorders were sporadic and were characterized by a progressive ataxia of gait, parkinsonism, and a mild terminal dementia. In a recent autoradiographic study of MSA, $\mathrm{GABA}_{\mathrm{A}}$ binding sites were unchanged in the molecular layer but significantly decreased in the granule cell layer [57]. $\mathrm{BDZ}$ binding and $\mathrm{GABA}_{\mathrm{B}}$ binding were unchanged in both layers. In the dentate nucleus, $\mathrm{GABA}_{\mathrm{A}}$ binding and $\mathrm{BDZ}$ binding were significantly increased.

Neurochemical studies of postmortem brain tissue in DOPCA demonstrated several different biochemical profiles of amino acid levels $[58,59]$. Most of these profiles include decreased glutamate, aspartate, and GABA levels in the cerebellar cortex and decreased GABA levels in the dentate nucleus. These profiles probably represent differences in the degree of degeneration of particular types of neurons among various cases of OPCA [60].

The results of three studies of $\mathrm{GABA}_{\mathrm{A}} / \mathrm{BDZ}$ receptor binding in OPCA have been reported $[56,61,62]$, and they are conflicting. Using $\left[{ }^{3} \mathrm{H}\right]$ flunitrazepam as a ligand, Kish and associates [62] found that BDZ receptor binding was either normal or slightly elevated in the cerebellar cortex. Binding in the deep cerebellar nuclei was not studied. As mentioned already, Whitehouse and coworkers [56] found that BDZ re- 
ceptor binding was unchanged in the cerebellar cortex, but increased in the dentate nucleus. Using in vitro postmortem autoradiography with $\left[{ }^{3} \mathrm{H}\right]$ flunitrazepam and $\left[{ }^{3} \mathrm{H}\right] \mathrm{GABA}$, Albin and Gilman [63] found a marked decrease of binding in the cerebellar cortex of 4 patients with OPCA. The reason the findings of Albin and Gilman [63] are different from those of Kish and associates [62] and Whitehouse and coworkers [56] may relate to biochemical differences between patients, probably because of variations in the degree of degeneration of the various types of neurons in the cerebellar cortex [58-60].

The principal conclusion from the present study is that $\mathrm{BDZ}$ receptors are largely preserved in the cerebellar cortex, brainstem, and basal ganglia in MSA, sOPCA, and DOPCA despite the known degenerative changes in many of these structures in these disorders. This finding is potentially important therapeutically, since it indicates that pharmacological interventions focused on $\mathrm{GABA}_{\mathrm{A}} / \mathrm{BDZ}$ receptors might offer some hope of alleviating the cerebellar and extrapyramidal symptoms of these disorders.

These investigations were supported in part by National Institutes of Health grants NS 15655, NS 24896, AG 08671, and AA 07378.

We thank the personnel of the PET/Cyclotron Center of the Division of Nuclear Medicine for production of the PET isotopes and acquisition of the scans, and Dr David Kuhl for his assistance.

\section{References}

1. Haefely WE. Pharmacology of the benzodiazepine receptor. Eur Arch Psychiatr Neurol Sci 1989;238:294-301

2. Matsumoto RR. GABA receptors: are cellular differences reflected in function? Brain Res Rev 1989;14:203-225

3. Olsen RW, McCabe RT, Wamsley JK. GABA A receptor subtypes: autoradiographic comparison of $\mathrm{GABA}$, benzodiazepine, and convulsant binding sites in the rat central nervous system. J Chem Neuroanat 1990;3:59-76

4. Haefely WE. The GABA-benzodiazepine interaction fifteen years later. Neurochem Res 1990;15:169-174

5. Enna SJ, Snyder SH. Influences of ions, enzymes and detergents on gamma-aminobutyric acid receptor binding in synaptic membranes of rat brain. Mol Pharmacol 1977;13:442-453

6. Olsen RW, Bergman MO, Van Ness PC, et al. Gamma-aminobutyric acid receptor binding in mammalian brain: heterogeneity of binding sites. Mol Pharmacol 1981;19:217-227

7. Olsen RW, Snowhill EW, Wamsley JK. Autoradiographic localization of low affinity GABA receptors with $\left[{ }^{3} \mathrm{H}\right]$ bicuculline methochloride. Eur J Pharmacol 1984;99:247-248

8. Palacios JM, Wamsley JK, Kuhar MJ. High affinity GABA receptors: autoradiographic localization. Brain Res 1981;222: 285-307

9. Palacios JM, Young WS III, Kuhar MJ. Autoradiographic localization of gamma-aminobutyric acid (GABA) receptors in the rat cerebellum. Proc Natl Acad Sci USA 1980;77:670-674

10. Olsen RW. GABA-benzodiazepine-barbiturate receptor interactions. J Neurochem 1981;37:1-13

11. McCabe RT, Wamsley JK. Autoradiographic localization of subcomponents of the macromolecular GABA receptor complex. Life Sci 1986;39:1937-1945
12. Mazière $M$, Hantraye $P$, Prenant $C$, et al. Synthesis of RO 1788 with ${ }^{11} \mathrm{C}$ : a specific radioligand for the in vivo study of central benzodiazepine receptors by positron emission tomography. Int J Appl Radiat Isot 1984;35:973-976

13. Koeppe RA, Holthoff VA, Frey KA, et al. Comparmental analysis of $\left[{ }^{11} \mathrm{C}\right]$ flumazenil kinetics for the estimation of ligand transport rate and receptor distribution using positron emission tomography. J Cereb Blood Flow Metab 1991;11:735-744

14. Frey KA, Holthoff VA, Koeppe RA, et al. Parametric in vivo imaging of benzodiazepine receptor distribution in human brain. Ann Neurol 1991;30:663-672

15. Quinn N. Multiple system atrophy-the nature of the beast J Neurol Neurosurg Psychiatry 1989;(special suppl):78-89

16. Quinn N, Marsden CD. The motor disorder of multiple system atrophy. J Neurol Neurosurg Psychiatry 1993;56:1239-1242

17. Polinsky RJ. Multiple system atrophy. Clinical aspects, pathophysiology and treatment. Neurol Clin 1984;2:487-498

18. Oppenheimer DR, Esiri MM. Diseases of the basal ganglia, cerebellum and motor neurons. In: Hume Adams J, Duchen LW, eds. Greenfield's neuropathology. Sth ed. London: Edward Arnold, 1992:988-1045

19. Kume A, Takahashi A, Hashizume Y, Asai J. A histometrical and comparative study on Purkinje cell loss and olivary nucleus cell loss in multiple system atrophy. J Neurol Sci 1991;101: 178-186

20. Fearnley JM, Lees AJ. Striatonigral degeneration. A clinicopathological study. Brain 1990;113:1823-1842

21. Gibb WRG. Accuracy in the clinical diagnosis of parkinsonian syndromes. Postgrad Med J 1988;64:345-351

22. Borit A, Rubinstein LJ, Urich $\mathrm{H}$. The striatonigral degenerations: putaminal pigments and nosology. Brain 1975;98:101112

23. Takei Y, Mirra SS. Striatonigral degeneration: a form of multiple system atrophy with clinical parkinsonism. In: Zimmerman HM, ed. Progress in neuropathology. New York: Grune \& Stratton, 1973:217-251

24. Cohen J, Low P, Fealey R, et al. Somatic and autonomic function in progressive autonomic failure and multiple system atrophy. Ann Neurol 1987;22:692-699

25. Spokes EGS, Bannister R, Oppenheimer DR. Multiple system atrophy with autonomic failure. J Neurol Sci 1979;43:59-82

26. Sung JH, Mastri AR, Segal E. Pathology of Shy-Drager syndrome. J Neuropathol Exp Neurol 1979;38:353-368

27. Bannister R, Oppenheimer DR. Degenerative diseases of the nervous system associated with autonomic failure. Brain 1972; 95:457-474

28. Papp MI, Khan JE, Lantos PL. Glial cytoplasmic inclusions in the CNS of patients with multiple system atrophy (striatonigral degeneration, olivopontocerebellar atrophy and Shy-Drager syndrome). J Neurol Sci 1989;94:79-100

29. Nakazato $Y$, Yamazaki $\mathrm{H}$, Hirato J, et al. Oligodendroglial microtubular tangles in olivopontocerebellar atrophy. J Neuropathol Exp Neurol 1990;49:521-530

30. Kato S, Nakamura H, Hirano A, et al. Argyrophilic ubiquinated cytoplasmic inclusions in Leu-7-positive glial cells in olivopontocerebellar atrophy (multiple system atrophy). Acta Neuropathol (Berl) 1991;82:488-493

31. Mochizuki A, Mizusawa $\mathrm{H}$, Ohkoshi $\mathrm{N}$, et al. Argentophilic intracytoplasmic inclusions in multiple system atrophy. J Neurol 1992;239:311-316

32. Papp MI, Lantos PL. The distribution of oligodendroglial inclusions in multiple system atrophy and its relevance to clinical symptomatology. Brain 1994;117:235-243

33. Kato $\mathrm{S}$, Nakamura $\mathrm{H}$. Cytoplasmic argyrophilic inclusions in neurons of pontine nuclei in patients with olivopontocerebellar atrophy: immunohistochemical and ultrastructural studies. Acta Neuropathol (Berl) 1990;79:584-594 
34. Papp MI, Lantos PL. Accumulation of tubular structures in oligodendroglial and neuronal cells as the basic alteration in multiple system atrophy. J Neurol Sci 1992;107:172-182

35. Lantos PL, Papp MI. Cellular pathology of multiple system atrophy: a review. J Neurol Neurosurg Psychiatry 1994;57:129133

36. Eadie MJ. Olivo-ponto-cerebellar atrophy (Dejerine-Thomas type). In: Vinken PJ, Bruyn GW, eds. Handbook of clinical neurology. Amsterdam: North-Holland, 1975:415-431

37. Eadie MJ. Olivo-ponto-cerebellar atrophy (Menzel type). In: Vinken PJ, Bruyn GW, eds. Handbook of clinical neurology. Amsterdam: North-Holland, 1975:433-449

38. Berciano J. Olivopontocerebellar atrophy. A review of 117 cases. J Neurol Sci 1982;53:253-272

39. Duvoisin RC. An apology and an introduction to the olivopontocerebellar atrophies. In: Duvoisin RC, Plaitakis A, eds. The olivopontocerebellar atrophies. New York: Raven, 1984:5-12

40. Harding A. 'Idiopathic' late onset cerebellar ataxia. In: Harding A, ed. The hereditary ataxias. Boston: Butterworths, 1984:166173

41. Durr A, Chneiweiss $\mathrm{H}$, Khati $\mathrm{C}$, et al. Phenotypic variability in autosomal dominant cerebellar ataxia type $I$ is unrelated to genetic heterogeneity. Brain 1993;116:1497-1508

42. Koeppen AH, Baron KD. The neuropathology of olivopontocerebellar atrophy. In: Duvoisin RC, Plaitakis A, eds. The olivopontocerebellar atrophies. New York: Raven, 1984:13-38

43. Koeppen $A H$. The Purkinje cell and its afferents in human hereditary ataxia. J Neuropathol Exp Neurol 1991;50:505-514

44. Ferrer I, Genís D, Dávalos A, et al. The Purkinje cell in olivopontocerebellar atrophy. A Golgi and immunocytochemical study. Neuropathol Appl Neurobiol 1994;20:38-46

45. Gilman S, Markel DS, Koeppe RA, et al. Cerebellar and brainstem hypometabolism in olivopontocerebellar atrophy detected with positron emission tomography. Ann Neurol 1988:24:223230

46. Rosenthal G, Gilman S, Koeppe RA, et al. Motor dysfunction in olivopontocerebellar atrophy is related to cerebral metabolic rate studied with positron emission tomography. Ann Neurol 1988;24:414-419

47. Kluin KJ, Gilman S, Markel DS, et al. Speech disorders in olivopontocerebellar atrophy correlate with positron emission tomography findings. Ann Neurol 1988;23:547-554

48. Savoiardo M, Strada L, Girotti F, et al. MR imaging in progressive supranuclear palsy and Shy-Drager syndrome. J Comput Assist Tomogr 1989;13:555-560

49. Savoiardo M, Strada L, Girotti F, et al. Olivopontocerebellar atrophy: MR diagnosis and relationship to multisystem atrophy Radiology 1990;174:693-696

50. Brooks DJ. Reply to the diagnosis of multiple system atrophy by T Caraceni. Ann Neurol 1991;29:690

51. McLeod JG, Tuck RR. Disorders of the autonomic nervous system: part 2. Investigation and treatment. Ann Neurol 1987; $21: 519-529$

52. Holthoff VA, Koeppe RA, Frey KA, et al. Differentiation of radioligand delivery and binding in the brain: validation of a two-compartment model for $\left[{ }^{11} \mathrm{C}\right]$ flumazenil. J Cereb Blood Flow Metab 1991;11:745-752

53. Gilman S, Koeppe RA, Junck L, et al. Patterns of cerebral glucose metabolism detected with PET differ in multiple system atrophy and olivopontocerebellar atrophy. Ann Neurol 1994; $36: 166-175$

54. Hochberg Y, Tamhane AC. Muitiple comparison procedures. New York: Wiley, 1987:30

55. Shinotoh $\mathrm{H}$, Tateno $\mathrm{Y}$, Hirayama $\mathrm{K}$. Benzodiazepine receptors in spino-cerebellar degeneration studied with positron emission tomography. Clin Neurol 1988;28:437-446

56. Whitehouse PJ, Muramoto O, Troncoso JC, Kanazawa I. Neurotransmitter receptors in olivopontocerebellar atrophy: an autoradiographic study. Neurology 1986;36:193-197

57. Price RH, Albin RI, Sakurai SY, et al. Cerebellar excitatory and inhibitory amino acid receptors in multiple system atrophy. Neurology 1993;43:1323-1328

58. Perry TL. Neurotransmitter abnormalities in dominantly inherited olivopontocerebellar atrophies. Ital J Neurol Sci 1984; (suppl 4):79-89

59. Perry TL. Four biochemically different types of dominantly inherited olivopontocerebellar atrophy. In: Duvoisin RC, Plaitakis A, eds. The olivopontocerebellar atrophies. New York: Raven, 1984:205-216

60. Kanazawa I, Rwak S, Hidenao S, et al. Density in the cerebellar system in olivopontocerebellar atrophy and cortical cerebellar atrophy. J Neurol Sci 1985;71:193-208

61. Kish SJ, Perry TL, Hornykiewicz O. Increased GABA receptor binding in dominantly-inherited cerebellar ataxias. Brain Res 1983;269:370-373

62. Kish SJ, Perry TL, Hornykiewicz O. Benzodiazepine receptor binding in cerebellar cortex: observations in olivopontocerebellar atrophy. J Neurochem 1984;42:466-469

63. Albin RL, Gilman S. Autoradiographic localization of inhibitory and excitatory amino acid neurotransmitter receptors in human normal and olivopontocerebellar atrophy cerebellar cortex. Brain Res 1990;522:37-45 